\title{
Myocardial infarction, aortitis and celiac trunk inflammation in giant cell arteritis: a case report
}

\begin{abstract}
We report a rare case of a 62-year-old woman admitted for investigation of unexplained inflammatory syndrome. Her atypical clinical presentation included a lack of headache and systemic signs. Computed tomography scan showed thoracic, abdominal aortitis and celiac trunk inflammation. A temporal artery biopsy was consistent with giant cell arteritis (GCA). She was treated with corticosteroids with a good response. Her unusual clinical presentation highlights the importance of considering GCA in the causes of aortitis and myocardial infarction in patients over 50 years, where prompt corticosteroid treatment results in satisfactory outcomes.
\end{abstract}

Retrosternal pain appeared on 7th day of hospitalization. An electrocardiogram revealed sinus rhythm of 75 beats/minute and ST-segment elevations in leads V1 to V5. Laboratory tests showed increased total plasma creatine kinase activity $561 \mathrm{U} / 1$ (normal range 25-195 $\mathrm{U} / \mathrm{l}$ ), and the blood troponin I level rose to $0.57 \mu \mathrm{g} / 14$ hour later (normal values $<0.1 \mu \mathrm{g} / \mathrm{l}$ )

Because coronary vasculitis was suspected, intravenous administration of unfractioned heparin and acetylsalicylic acid were initiated and resulting in a relief of the chest pain and resolution of ST segment changes on electrocardiogram within 24 hours. Echocardiogram revealed hypokinesia of the apical segment of the anterior wall, as well as diminished left ventricular ejection fraction of approximately $45 \%$. Coronary angiography performed later revealed no significant lesion.

On discharge, corticosteroid therapy was continued and tapered 6 weeks later. The patient did not experience further episodes of chest pain or other cardiovascular complications during 1year of follow- up.
Volume 4 Issue 6 - 2017

\author{
Yosra Cherif,' Olfa Berriche, ${ }^{2}$ Sonia \\ Hammami ${ }^{3}$ \\ 'Department of Internal Medicine CHU F Bourguiba Monastir, \\ Tunisia \\ 'UR03/ ES-08' Nutrition Humaine et Desordres Metaboliques, \\ Tunisia \\ ${ }^{3}$ Faculty of medicine, University of Monastir, Tunisia
}

Correspondence: Olfa Berriche, Department of Internal Medicine CHU F Bourguiba Monastir,Tunisia;

Email: olfaberriche@gmail.com

Received: March 09, 2017 | Published: June 02, 2017

\section{Introduction}

Few cases of acute coronary events are related to non atherosclerotic processes, such as coronary embolism, dissection, congenital abnormalities and vasculitis. ${ }^{1}$ Giant cell arteritis (GCA) affects mainly arteries of large and medium caliber, especially the extra-cranial branches of the carotids. It may rarely affect coronary vessels. ${ }^{1-12}$

Although easily identified if a patient presents with typical symptoms, this diagnosis may often be complicated if the condition shows atypical clinical features.

We report a case of a woman diagnosed with aortitis, celiac trunk inflammation and an acute myocardial infarction due to coronary vasculitis secondary to GCA.

\section{Case report}

A 62-year-old woman with 4 day history of moderate headaches was admitted to the department of Internal Medicine for investigation. On admission, her pulse rate 74 beats/min, arterial blood pressure was $110 / 70 \mathrm{mmHg}$, symmetrical over upper and lower extremity arteries. All peripheral pulses were present. The remaining physical examination was normal.

Laboratory tests showed hemoglobin $8.5 \mathrm{~g} / \mathrm{dl}$, white blood cell count $10250 / \mathrm{mm}^{3}$, erythrocyte sedimentation rate was $124 \mathrm{~mm}$ hour, and level of C - reactive protein was $59 \mathrm{mg} / \mathrm{l}$, serum fibrinogen concentration $5.4 \mathrm{~g} / \mathrm{l}$, serum albumin level $36 \mathrm{~g} / \mathrm{l}$ and an increased serum globulin $16.8 \mathrm{~g} / \mathrm{l}$. Other laboratory tests, especially the lipid profile were within the normal range. Microbiological cultures were unremarkable. A computed tomography scan revealed an ascending aortic thickening of the thoracic, abdominal aortic wall and celiac trunk with periaortic inflammation extending to the iliac arteries (Figure 1). It also disclosed bilateral subclavian artery stenosis. There was no aneurysm or dissection.

Given that headaches wosened, became constant and unremitting, temporal artery biopsy was performed and revealed features consistent with diagnosis of GCA. A high regimen of corticosteroid therefore was initiated.

Retrosternal pain appeared on 7th day of hospitalization. An electrocardiogram revealed sinus rhythm of 75 beats/minute and ST-segment elevations in leads V1 to V5.Laboratory tests showed increased total plasma creatine kinase activity $561 \mathrm{U} / 1$ (normal range 25-195 U/l), and the blood troponin I level rose to $0.57 \mu \mathrm{g} / 14$ hour later (normal values $<0.1 \mu \mathrm{g} / \mathrm{l}$ ).

Because coronary vasculitis was suspected, intravenous administration of unfractioned heparin and acetylsalicylic acid were initiated and resulting in a relief of the chest pain and resolution of ST segment changes on electrocardiogram within 24hours. Echocardiogram revealed hypokinesia of the apical segment of the anterior wall, as well as diminished left ventricular ejection fraction of 
approximately $45 \%$. Coronary angiography performed later revealed no significant lesion.

On discharge, corticosteroid therapy was continued and tapered 6 weeks later. The patient did not experience further episodes of chest pain or other cardiovascular complications during lyear of follow- up.

\section{Discussion}

Myocardial infarction may result from nonatherosclerotic coronary processes such as coronary embolism, spasm, dissection, vasculitis, steroid treatment and Congenital coronary anomalies. ${ }^{1}$ Several vasculitis and autoimmune diseases such as Behçet's disease, ployarteritisnodosa, Takayasu disease, GCA may affect coronary vessels. ${ }^{1}$

GCA is an inflammatory disease affecting the aorta, its major branches and the temporal and vertebral arteries. It typically affects elderly patients.

According to American College of Rheumatology (ACR) criteria, ${ }^{12}$ our patient presented with at least four (age, headache, erythrocyte sedimentation rate greater than $50 \mathrm{~mm} /$ hour, documented lesion in the temporal artery) of five required criteria. It is difficult to distinguish between inflammatory, iatrogenic and atherosclerotic arterial injury face of an old patient with GCA. Clinical features, angiography and response to corticosteroid therapy provided strong evidence that coronary vasculitis was attributable to GCA in our patient.

Few cases with an isolated coronary lesion in GCA were recorded in literature. ${ }^{2,3}$ The present case is of special interest because the coronary arteritis occured within one week of the onset of corticosteroid therapy in a patient without cardiovascular risk factor with GCA.

Coronary arteries are involved in few cases of GCA..$^{2-4,6,8,10}$

The pathogenesis of GCA myocarditis speculate a diffuse vasculitis of coronary vessels. ${ }^{2,4,6,9}$ In addition, the disease process enhances and increases atherosclerosis and platelet aggregation. ${ }^{9}$ Inflammatory thickening of coronary arteries may lead to their occlusion and weakening of the vessel wall.

Another feared complication is aortitis. About $20 \%$ of patients with GCA develop aortitis, ${ }^{10-12}$ that may sometimes reveal the diagnosis. Many of these cases develop aortic aneurysms with a high risk of aortic rupture. ${ }^{11,12}$ The diagnosis of aortitis in our patient was fortuitous, when she was screened for the unexplained biological inflammation. Despite the large extent of aortitis scanning the thoracic, abdominal aorta and celiac trunk, our patient showed no complaint or nonspecific features. Besides, few cases of celiac vasculitis in GCA were reported. ${ }^{13}$

The atypical clinical presentation in our patient included a lack of headache and fever, but she exhibited signs of marked inflammatory syndrome. Her unusual presentation highlights the importance of considering GCA in the causes of aortitis, ${ }^{11,12}$ where prompt corticosteroid and immunosuppressive therapy to improve clinical outcomes in GCA..$^{7-10}$ It must be stressed, however, that there are no guidelines to treat coronary lesions related to GCA. The alterations of the wall of the vessels may result in a high rate of re-stenosis after invasive procedures. ${ }^{10}$ The short-term prognosis of GCA myocardial infarction is poor, and it may increase mortality if corticosteroid therapy is delayed. . $^{5,6,9,12}$

In our case, we did not perform coronary angioplasty or stent implantation. Instead, we maintained corticosteroid treatment with acetylsalicylic acid.
Our observation further shows that GCA might be borne in mind as a cause of acute coronary syndrome and aortitisin patients over 50 years with extracardiac symptoms and biological inflammation. Temporal artery biopsy should be performed in patients over age 50 years with myocardial infarction; aortitis when biological inflammation is proved, even in the absence of suggestive clinical signs (Figure 1).

A

B

C

Figure I (A) A computed tomography scan revealed an ascending aortic thickening of the thoracic, abdominal aortic wall (B) and celiac trunk (C) with

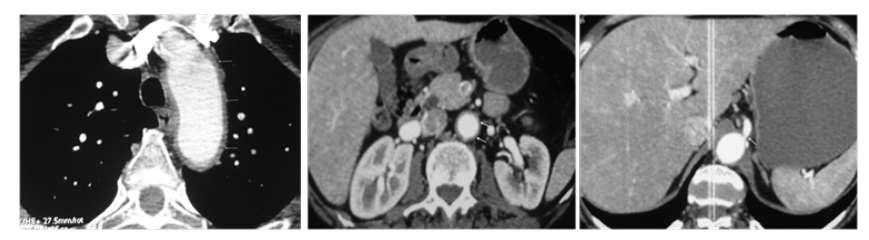

aortic inflammation extending to the iliac arteries.

\section{Acknowledgements}

None.

\section{Conflicts of interest}

The author declares there is no conflict of interest.

\section{References}

1. Mirza A. Myocardial infarction resulting from nonatherosclerotic coronary artery diseases. Am J Emergency Med. 2003;(21):578-584.

2. Teixeira A, Capitaine E, Congy F. Myopericarditis during Horton disease. Rev Med Interne. 2003;24(3):189-194.

3. Lin LW, Wang SS, Shun CT. Myocardial infarction due to giant cell arteritis: a case report and literature review. Kaohsiung $\mathrm{J} \mathrm{Med} \mathrm{Sci.}$ $2007 ; 23(4): 195-198$.

4. Pugnet G, Pathak A, Dumonteil N, et al. Giant cell arteritis as a cause of acute myocarditis in the elderly. $J$ Rheumatol. 2011;38(11):2497.

5. Agard C, Espitia O, Néel A. Prognosis of giant cell arteritis. Presse Med. 2012;41:966-974.

6. Morris CR, Scheib JS. Fatal myocardial infarction resulting from coronary arteritis in a patient with polymyalgia rheumatica and biopsyproved temporal arteritis. A case report and review of the literature. Arch Intern Med. 1994;154(10):1158-1160.

7. Andrade J, Al Ali A, Saw J, et al. Acute stent thrombosis in a patient with giant cell arteritis. Can J Cardiol. 2008;24(4):e25-e26.

8. Godoy P, Araujosde A, Paulino E, et al. Coronary giant cell arteritis and acute myocardial infarction. Arq Bras Cardiol. 2007;88(4):e84-e87.

9. Karger B, Fechner G. Sudden death due to giant cell coronary arteritis. Int J Legal Med. 2006;120:377-379.

10. Wheeler G, Kropf J, Jeanmonod R. Vasculitis presenting with myocardial infarction, stroke, and aortitis in a middle-aged man. J Emerg Med. 2012;43(4):655-658.

11. Josselin-Mahr L, El Hessen TA, Toledano C, et al. Inflammatory aortitis in giant cell arteritis. Presse Med. 2013;42(2):151-159.

12. Calvo-Romero JM. Giant cell arteritis. Postgrad Med J. 2003;79(935):511-515.

13. Evans DC, Murphy MP, Lawson JH. Giant cell arteritis manifesting as mesenteric ischemia. $J$ Vasc Surg. 2005;42(5):1019-1022. 